\title{
Toxicité sérotoninergique résultant d'une interaction médicamenteuse entre le bleu de méthylène et les inhibiteurs de la recapture de la sérotonine
}

\author{
par Annie Charbonneau
}

\section{RÉSUMÉ}

Contexte : Le bleu de méthylène est employé en pratique pour diverses raisons médicales. Des données récentes ont évoqué une interaction potentielle avec les inhibiteurs de la recapture de la sérotonine, pouvant conduire à une toxicité sérotoninergique.

Objectif : Décrire le risque de toxicité sérotoninergique associé à l'interaction entre le bleu de méthylène et les inhibiteurs de la recapture de la sérotonine.

Sources de l'information : Les publications pertinentes ont été ciblées systématiquement au moyen des moteurs de recherche MEDLINE (1946 au 21 mars 2013) et Embase (1974 à 2013, semaine 11) en utilisant les mots clés suivants : methylene blue, methylthioninium, monoamine oxidase inhibitors, serotonin reuptake inhibitors et serotonin syndrome. Aucune restriction touchant l'indication du bleu de méthylène ou la langue n'a été appliquée. Les références des publications ont également été analysées.

Sélection des études et extraction des données : Dix-huit études de cas et deux séries de cas systématiques ont été sélectionnées. Aucune étude clinique aléatoire n’a encore été publiée.

Synthèse des résultats : La première étude de cas à avoir soupçonné une interaction entre le bleu de méthylène et les inhibiteurs de la recapture de la sérotonine est parue en 2003. Dix-sept autres études de cas décrivant le même type d'interaction ont par la suite été rapportées. Les deux séries de cas ont regroupé les données de quelques 325 parathyroïdectomies où le bleu de méthylène avait été employé comme agent colorant. Les 17 patients qui ont présenté une toxicité du système nerveux central prenaient tous en période préopératoire des inhibiteurs de recapture de la sérotonine.

Conclusion : Lorsqu'il est administré en concomitance avec des inhibiteurs de recapture de la sérotonine, le bleu de méthylène peut conduire à une toxicité sérotoninergique à une dose aussi faible que $0,7 \mathrm{mg} / \mathrm{kg}$. En effet, il posséderait des propriétés inhibitrices de la monoamine oxidase A. Des précautions doivent être prises pour éviter cette interaction.

Mots clés : bleu de méthylène, méthylthioninium, inhibiteurs de monoamine oxidase, inhibiteurs de la recapture de la sérotonine, syndrome sérotoninergique

J Can Pharm Hosp 2013;66(4):241-52

\begin{abstract}
Background: Methylene blue is used in medical practice for various reasons. Recent findings point to a potential interaction with serotonin reuptake inhibitors (SRIs) that could lead to serotonergic toxicity.
\end{abstract}

Objective: To describe the risk of serotonergic toxicity associated with the interaction between methylene blue and SRIs.

Data sources: Relevant publications were searched systematically via MEDLINE (1946 to March 21, 2013) and Embase (1974 to 2013, week 11) with the following search terms: "methylene blue", "methylthioninium", "monoamine oxidase inhibitors", "serotonin reuptake inhibitors", and "serotonin syndrome". No restrictions were applied in relation to the indication for methylene blue or the language of publication. The reference lists of identified articles were also searched.

Study selection and data extraction: Eighteen case reports and 2 case series were identified for inclusion. To date, no randomized controlled trials have been published.

Data synthesis: The first case report indicating suspicion of an interaction between methylene blue and SRIs was published in 2003. Seventeen other case reports describing the same type of interaction have been published since then. The 2 case series provided data from about 325 parathyroidectomies in which methylene blue was used for staining. The 17 patients who experienced central nervous system toxicity were all taking SRIs in the preoperative period.

Conclusion: When administered in combination with SRIs, methylene blue may lead to serotonergic toxicity at doses as low as $0.7 \mathrm{mg} / \mathrm{kg}$. Methylene blue would seem to have monoamine oxidase A inhibitory properties. Precautions should be taken to avoid this interaction.

Keywords: methylene blue, methylthioninium, monoamine oxidase inhibitors, serotonin reuptake inhibitors, serotonin syndrome

[Publisher's translation] 


\section{INTRODUCTION}

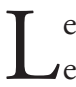
e bleu de méthylène (BM) ou chlorure de méthylthioninium est un dérivé des phénothiazines; il est couramment employé en pratique pour le traitement de la méthémoglobinémie, de l'encéphalopathie provoquée par l'ifosfamide et du syndrome vasoplégique. Il est également utilisé comme agent adjuvant lors de procédures chirurgicales ${ }^{1-4}$. Cependant, aucune de ces indications n'est officiellement approuvée par la Food and Drug Administration (FDA) des États-Unis présentement ${ }^{5}$. Quant à Santé Canada, aucune monographie du produit n'est disponible sur son site officiel.

Au cours de la dernière décennie, des données de la littérature scientifique ont évoqué un risque de toxicité sérotoninergique lorsque le $\mathrm{BM}$, sans égard pour l'indication thérapeutique, est employé chez des patients prenant des inhibiteurs de recapture de la sérotonine (IRS). Cette revue systématique présentera tout d'abord une brève description des différentes doses thérapeutiques du BM ainsi que de la toxicité sérotoninergique avant de procéder à une analyse approfondie de l'interaction entre le BM et les IRS.

\section{MÉTHODOLOGIE}

Une revue systématique de la littérature a été effectuée au moyen des moteurs de recherche MEDLINE (1946 au 21 mars 2013) et Embase (1974 au 2013, semaine 11) sans aucune restriction touchant la langue ou l'indication du BM. La liste des mots-clés ayant servi à la recherche sont les suivants : methylene blue, methylthioninium, monoamine oxidase inhibitors, serotonin reuptake inhibitors et serotonin syndrome. Une analyse des références des articles identifiés a permis d'extraire toute information supplémentaire. Pour être retenues, les publications devaient répondre aux critères suivants : les sujets à l'étude devaient avoir reçu un IRS et développé une toxicité sérotoninergique ou une toxicité du système nerveux central (SNC) à la suite de l'administration du BM.

\section{Doses de BM employées en pratique}

Plusieurs posologies sont disponibles en ce qui a trait à l'administration du BM. Pour le traitement de la méthémoglobinémie, la dose usuelle de $\mathrm{BM}$ visant à réduire la méthémoglobine en hémoglobine est de 1 à $2 \mathrm{mg} / \mathrm{kg}$ par voie intraveineuse (IV $)^{1,4}$. Quant au traitement du syndrome vasoplégique, la dose thérapeutique est de $2 \mathrm{mg} / \mathrm{kg}$, l'infusion se faisant généralement sur une période de 20 minutes. En effet, le BM bloque la guanylate cyclase, inhibant ainsi l'effet vasodilatateur de l'oxyde nitrique, ce qui a pour effet d'augmenter la tension artérielle $e^{1,3,6}$. Le BM peut aussi être administré à une dose de $50 \mathrm{mg}$ IV toutes les quatre heures jusqu'à la résolution des symptômes dans le traitement de l'encéphalopathie causée par l'ifosfamide ${ }^{1,2}$.
Dans le cadre de procédures chirurgicales, le BM peut grandement faciliter la localisation de la structure à réséquer afin de réduire les dommages nerveux ${ }^{7-10}$. Bien qu'aucune étude prospective n'appuie son utilisation, le BM est employé depuis 1971 lors de parathyroïdectomies ${ }^{7,11}$. Une seule dose IV de 5 à $7,5 \mathrm{mgkg}$ diluée dans $500 \mathrm{~mL}$ de solution normale saline est généralement administrée en infusion continue après le début de l'anesthésie ${ }^{10,12,13}$. Il en résulte alors une coloration bleu foncé des adénomes alors que les autres structures normales de la parathyroïde sont colorées à un degré moindre. Les propriétés colorantes du BM sont aussi utiles pour la résection de polypes cancéreux du côlon. Une quantité minimale de $1 \mathrm{~mL}$ de BM dosé à $1 \%$ est injectée localement dans les sous-muqueuses colorectales, délimitant ainsi une bulle bleutée prête à être excisée $^{14,15}$.

Le BM employé à des doses inférieures à $7 \mathrm{mgkg}$ est associé à peu d'effets indésirables. Cependant, des doses supérieures à $7 \mathrm{mgkg}$ seraient associées à une augmentation de cas de nausée, de douleur abdominale, de confusion et de méthémoglobinémie ${ }^{1,16,17}$.

\section{Physiopathologie de la toxicité sérotoninergique}

La toxicité sérotoninergique est la conséquence d'une surstimulation des récepteurs sérotoninergiques postsynaptiques dans le $\mathrm{SNC}$ et le système nerveux périphérique ${ }^{18}$. La gravité des cas de toxicité sérotoninergique serait directement proportionnelle à la concentration de sérotonine dans la fente synaptique $^{19}$. Pour le moment, la physiopathologie n'est pas encore complètement élucidée, mais on pense que les sousrécepteurs 5-HT2A contribueraient significativement à cette toxicité. Certains auteurs ont émis l'hypothèse que les sousrécepteurs 5-HT1A seraient également impliqués en augmentant la concentration intrasynaptique des agonistes sérotoninergiques, ce qui saturerait les autres sousrécepteurs ${ }^{20,21}$. Il semblerait que cette hypothèse ne tienne plus la route étant donné l'inefficacité des antagonistes des récepteurs 5-HT1A dans le traitement de la toxicité sérotoninergique. Dans certains cas, ceux-ci ont même été associés à une aggravation de la condition ${ }^{21}$.

Généralement, l'apparition des signes et symptômes d'une toxicité sérotoninergique est rapide. En effet, $60 \%$ d'entre eux surgissent lors des six premières heures après la surdose ou l'augmentation de dose d'un agent sérotoninergique à libération immédiate ${ }^{22,23}$. Toutefois, en présence d'un agent sérotoninergique à libération retardée, il y a un délai des pics de concentrations plasmatiques et, par conséquent, de l'apparition des signes et symptômes. Par exemple, le tableau est retardé de douze heures après une surdose de venlafaxine ${ }^{22}$.

Les surdoses d'inhibiteurs sélectifs de la recapture de la sérotonine (ISRS) contribuent à seulement $15 \%$ des cas de 
Tableau 1. Médicaments associés à la toxicité sérotoninergique ${ }^{18,21,22,27,28}$

\begin{tabular}{|c|c|}
\hline \multirow{2}{*}{$\begin{array}{l}\text { Classe de médicaments } \\
\text { Inhibiteurs de la recapture de } \\
\text { la sérotonine }\end{array}$} & Exemples \\
\hline & $\begin{array}{l}\text { Inhibiteurs sélectifs de la recapture de la sérotonine: } \\
\text { paroxétine, fluvoxamine, fluoxétine, sertraline, citalopram, } \\
\text { escitalopram } \\
\text { Inhibiteurs de la recapture de la sérotonine et de la } \\
\text { norépinéphrine : venlafaxine, duloxétine, desvenlafaxine } \\
\text { Antidépresseurs tricycliques : clomipramine et imipramine } \\
\text { seulement } \\
\text { Chlorphéniramine } \\
\text { Opioïdes : tramadol, fentanyl, méthadone, dextrométhorphan } \\
\text { Chlorphéniramine } \\
\text { Sibutramine } \\
\text { Millepertuis }\end{array}$ \\
\hline $\begin{array}{l}\text { Médicaments libérateurs } \\
\text { de sérotonine }\end{array}$ & $\begin{array}{l}\text { Méthylènedioxymethamphétamine (MDMA) } \\
\text { Cocaïne } \\
\text { Amphétamines } \\
\text { Méthamphétamines }\end{array}$ \\
\hline $\begin{array}{l}\text { Inhibiteurs de la monoamine } \\
\text { oxidase (IMAO) }\end{array}$ & $\begin{array}{l}\text { IMAO-A : moclobémide, linézolide } \\
\text { IMAO-B : sélégiline } \\
\text { IMAO-A+B : phénelzine, tranylcypromine }\end{array}$ \\
\hline Divers & $\begin{array}{l}\text { Lithium } \\
\text { Buspirone }\end{array}$ \\
\hline
\end{tabular}

toxicité sérotoninergique et sont considérées comme des toxicités d'intensité modérée. La majorité des toxicités importantes sont le résultat d'une administration concomitante de médicaments sérotoninergiques qui possèdent différents mécanismes d'action ${ }^{22,24,25}$. Trois classes de médicaments sont principalement en cause : les IRS, les agents libérateurs de sérotonine ainsi que les inhibiteurs de monoamine oxidase (IMAO), qui diminuent le métabolisme de la sérotonine ${ }^{23,26}$ (se référer au tableau 1). L'interaction médicamenteuse la plus décrite dans la littérature demeure sans contredit celle des ISRS et $\mathrm{IMAO}^{24}$.

Il est important de mentionner que la mirtazapine, le bupropion, la trazodone ainsi que tous les antidépresseurs tricycliques autres que la clomipramine et l'imipramine sont peu susceptibles, suite à une surdose ou une interaction avec un autre agent sérotoninergique, d'entraîner une toxicité sérotoninergique, vu leurs faibles propriétés sérotoninergiques ${ }^{27}$.

\section{Diagnostic de la toxicité sérotoninergique}

La toxicité sérotoninergique est généralement caractérisée par une triade clinique d'altération de l'état mental, de perturbation du système nerveux autonome et d'hyperactivité neuromusculaire ${ }^{18,21}$. Cependant, ces signes ne sont pas nécessairement simultanément présents dans tous les $\operatorname{cas}^{29}$. La toxicité sérotoninergique n'est pas un diagnostic d'exclusion, mais il n'existe pas non plus de tests de laboratoire qui lui sont spécifiques. Son diagnostic est uniquement établi sur une base clinique ${ }^{20,22,27}$.

Le diagnostic devrait suivre deux étapes : la détermination des signes cliniques compatibles avec la toxicité sérotonin- ergique puis l'évaluation de leur importance ${ }^{22}$. Les cas légers peuvent être associés à des signes peu spécifiques, tels la mydriase, des tremblements et la diaphorèse, tandis que les cas plus graves peuvent conduire au délirium, à l'hyperthermie et à la rigidité neuromusculaire, selon la concentration de sérotonine dans les fentes synaptiques ${ }^{20,22}$. Ainsi, il est préférable d'employer le terme toxicité sérotoninergique plutôt que syndrome sérotoninergique, car la toxicité sérotoninergique s'avère être une continuité d'effets indésirables grandissants ${ }^{30}$.

Plusieurs critères diagnostiques ont été suggérés au fil du temps. Les plus connus sont ceux de Sternbach et ceux de Hunter $^{22}$ (voir boîte 1). Ceux de Hunter sont les critères de prédilection pour le diagnostic de toxicité sérotoninergique, car ils sont plus précis (84\% versus $75 \%$ ) et plus spécifiques (97\% versus $96 \%$ ) que ceux de Sternbach. Ils ont été développés à la suite de l'étude d'un grand nombre de patients victimes de surdose d'agents sérotoninergiques et ont été par la suite validés par un département de toxicologie. Le critère-clé de Hunter est le clonus ${ }^{22,31}$.

\section{RÉSULTATS}

La figure 1 démontre le processus d'inclusion et d'exclusion des articles retenus lors de la stratégie de recherche. Deux études de cas et une série de cas ont été exclues pour les raisons suivantes : aucune mention de la prise d'IRS au moment de l'administration du $\mathrm{BM}$ à un patient qui présentait une complication neurologique, aucune mention d'IRS ni de toxicité sérotoninergique pour un autre patient qui avait reçu du $\mathrm{BM}$ et, finalement, aucune mention du BM reçu par trois 
Boîte 1. Critères de Hunter pour le diagnostic de la toxicité sérotoninergique ${ }^{31,32}$

En présence d'un agent sérotoninergique, rencontre d'au moins un des critères suivants :

1. Clonus spontané

2. Clonus inductible ET (agitation OU diaphorèse)

3. Clonus oculaire ET (agitation OU diaphorèse)

4. Tremblements ET hyperréflexie

5. Hypertonicité ET température supérieure à $38^{\circ} \mathrm{C}$ ET (clonus oculaire OU clonus inductible)

20 études de cas et 3 séries de cas identifiées à l'aide des moteurs de recherche MEDLINE et EMBASE

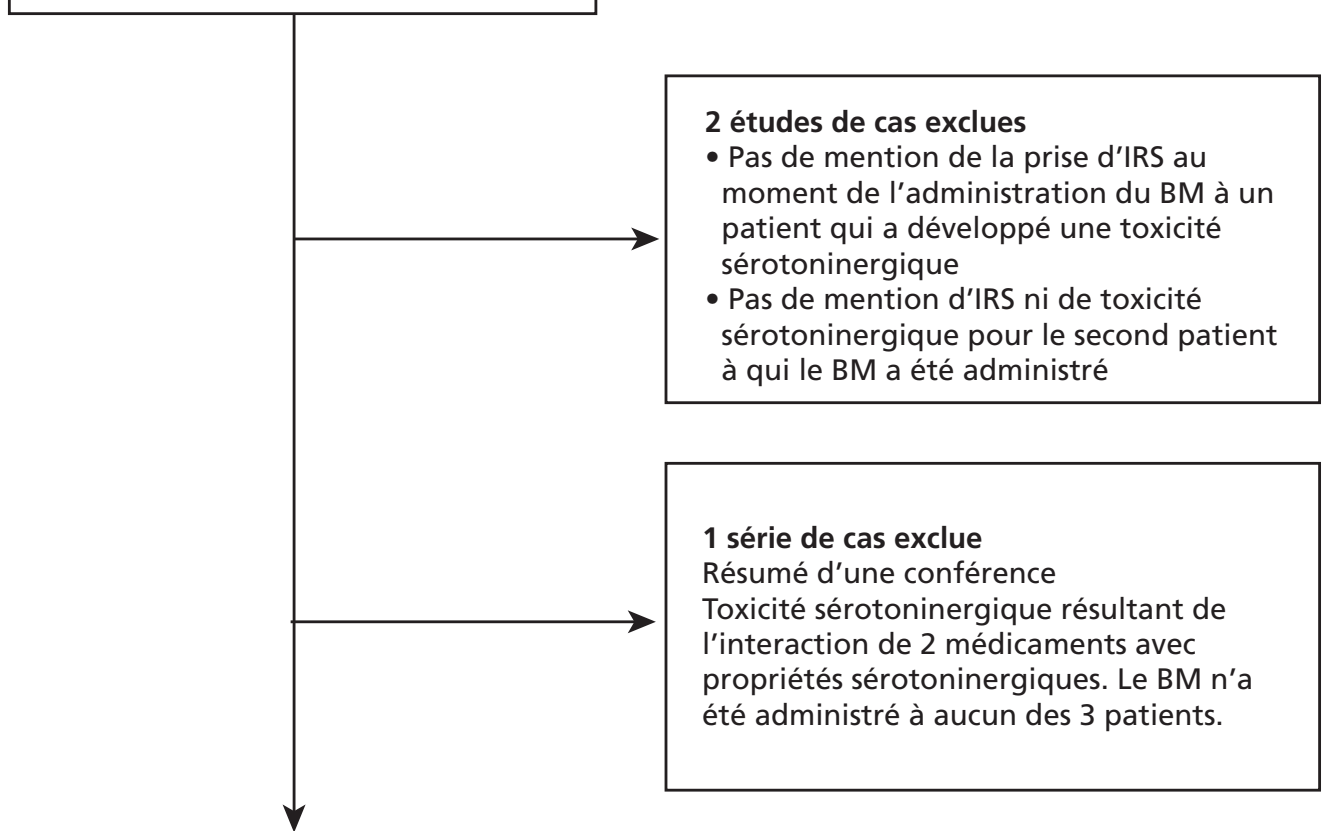

18 études de cas et 2 séries de cas incluses dans la revue systématique

Figure 1. Diagramme illustrant l'inclusion et les raisons d'exclusion des études. BM = bleu de méthylène, IRS = inhibiteur de la recapture de la sérotonine.

patients qui ont développé une toxicité sérotoninergique à la suite de l'interaction entre deux médicaments avec propriétés sérotoninergiques ${ }^{33-35}$. En tout, 18 études de $\operatorname{cas}^{13,36-53}$ ainsi que deux séries de $\operatorname{cas}^{54,55}$ ont été retenues pour réaliser cette revue systématique, puisque toutes décrivaient une toxicité sérotoninergique ou une toxicité du SNC résultant de l'interaction du BM avec des IRS ${ }^{13,36-55}$. Cependant, aucune étude observationnelle et aucune étude clinique aléatore n’ont encore été publiées.
C'est en 2003 qu'une toxicité du SNC liée à l'interaction du BM et d'un IRS a été évoquée pour la première fois dans la littérature scientifique. En effet, Martindale et Stedeford ${ }^{36}$ ont rapporté une toxicité intrinsèque du SNC associée au BM employé comme agent colorant dans le cadre d'une parathyroïdectomie. Aucun rapprochement avec la paroxétine prise par la patiente en période préopératoire n'a été effectué ${ }^{36}$. En 2006, Majithia et Stearns ${ }^{37}$ ont décrit une toxicité neurologique liée au $B M$ pouvant potentiellement être le 
résultat d'une interaction médicamenteuse entre le BM et un IRS. Toujours la même année, Rosenbaum ${ }^{38}$ ont précisé parmi leurs diagnostics différentiels la possibilité d'une toxicité sérotoninergique découlant de l'interaction entre le BM et un IRS. Par la suite, la publication de quinze autres études de cas et de deux séries de cas n'a fait que renforcer l'hypothèse d'une interaction entre le $\mathrm{BM}$ et les IRS pouvant conduire à une toxicité sérotoninergique.

Parmi l'ensemble des études de cas publiées jusqu'à présent, le BM a été administré pour la localisation de la glande parathyroïde dans le cadre de parathyroïdectomies dans 14 d'entre elles $(77,7 \%)$. Le BM a aussi été employé pour le traitement du syndrome vasoplégique (2 études de cas), pour la localisation de l'ostium urétéral (1 étude de cas) et pour le traitement de l'encéphalopathie causée par l'ifosfamide (1 étude de cas) ${ }^{43,50-52}$ (se référer au tableau 2). La dose de BM rapportée variait entre $0,7 \mathrm{mg} / \mathrm{kg}$ et $7,5 \mathrm{mg} / \mathrm{kg}$. Les dix-huit patients ont tous développé des signes et symptômes de toxicité du SNC en période postopératoire et plus précisément, pour la majorité d'entre eux, lors de la première heure après la fin de la chirurgie. Onze patients $(61 \%)$ ont nécessité une réintubation. Onze patients $(61 \%)$ ont vu la résolution des signes et symptômes lors des quatre premiers jours après l'administration du BM, ce qui correspond au temps d'élimination de la substance ${ }^{39}$. Six patients $(33 \%)$ ont vu leur état s'améliorer en une semaine. Un patient a dû avoir recours à la dialyse pour éliminer le $\mathrm{BM}^{46}$. Les dix-huit patients recevaient tous un IRS en période préopératoire. Quatorze d'entre eux (78\%) prenaient un ISRS, dont une patiente en combinaison avec la buspirone, un agoniste partiel du récepteur 5-HT1A, ainsi qu'une autre en combinaison avec le tramadol ${ }^{38,53,56}$. Deux autres patients $(11 \%)$ prenaient un inhibiteur de la recapture de la sérotonine et de la norépinéphrine (IRSN) (venlafaxine et duloxétine) au moment de la chirurgie, et deux patients (11\%) étaient traités avec la clomipramine, un antidépresseur tricyclique. Plusieurs diagnostics différentiels ont été évoqués dans les études de cas : méthémoglobinémie induite par le BM, encéphalopathie, réaction indésirable aux opiö̈des ou aux anesthésiques, tels le propofol, sevrage d'alcool, syndrome neuroleptique malin et toxicité sérotoninergique. L'hypothèse de méthémoglobinémie a été exclue dans sept d'entre elles en raison des niveaux de méthémoglobine inférieurs à $20 \%$. Sept auteurs (39 \%) ont évoqué une toxicité sérotoninergique potentielle parmi leurs diagnostics différentiels mais seulement trois d'entre eux ont explicitement cité les critères de Sternbach et Hunter dans leur discussion dans le but de confirmer ou de rejeter leur hypothèse $e^{40,42}$. Finalement, parmi l'ensemble des études de cas publiées à ce jour, sept d'entre elles (39\%) ont conclu à un diagnostic officiel de toxicité sérotoninergique ${ }^{41,43,44,50-53}$.

Deux séries de cas ont aussi rapporté une toxicité du SNC associée à l'utilisation du BM comme agent colorant ${ }^{54,55}$. Kartha et coll..$^{55}$ ont collecté les données de quelques 193 parathyroïdectomies effectuées entre 1998 et 2003. Une dose de $\mathrm{BM}$ de $7,5 \mathrm{mg} / \mathrm{kg}$ a été administrée lors de toutes ces interventions chirurgicales. Parmi ces patients, 12 d'entre eux $(6,2 \%)$ ont développé des signes et symptômes de toxicité du SNC en période postopératoire (intervalle de 1 à 11 heures). Il n'est pas surprenant de constater que tous ces patients prenaient un IRS en période préopératoire. Pour mesurer l'intensité de la toxicité, on l'a divisée en 12 paliers allant de la simple confusion jusqu'au délirium. Selon les auteurs, la toxicité du SNC se rapprochait davantage d'un état transitoire d'encéphalopathie métabolique toxique, tel que le suggérait l'électroencéphalogramme de quatre patients. La possibilité d'une toxicité sérotoninergique n'a aucunement été mentionnée dans leur discussion. La durée moyenne des signes et symptômes fut de 35,7 heures. De ces 12 patients, 11 ont récupéré sans aucune séquelle, et un patient est décédé des suites d'un arrêt cardiorespiratoire. Parmi les 181 patients n'ayant pas développé de toxicité, 16 (8,8 \%) prenaient un IRS. Les auteurs ont conclu qu'il fallait éviter d'utiliser du BM chez les patients prenant un IRS et qui devaient subir une parathyroïdectomie.

Sweet et Standiford ${ }^{54}$ ont compilé les données de 132 autres parathyroïdectomies survenues entre 2000 et 2006. Le $\mathrm{BM}$ a été administré à tous ces patients à une dose variant entre $3 \mathrm{mg} / \mathrm{kg}$ et $5 \mathrm{mg} / \mathrm{kg}$. Cinq patients (3,8\%) sur 132 se sont vu diagnostiquer une encéphalopathie postopératoire survenue dans un intervalle d'une à cinq heures après la fin de la chirurgie et reposant sur des signes et symptômes d'aphasie, de confusion, de désorientation, de léthargie et d'agitation d'intensité variable. Deux de ces patients ont été réintubés. Ces cinq patients prenaient tous un IRS (deux patients, de la fluoxétine; un patient, de l'escitalopram; et deux patients, de la venlafaxine). En deux à trois jours, l'encéphalopathie de ces cinq patients s'est complètement résorbée sans aucune intervention particulière. Parmi les 127 patients qui n'ont expérimenté aucune toxicité du SNC, 12 (9,4\%) prenaient un IRS avant la chirurgie. Les auteurs ont exclu les hypothèses d'effets indésirables secondaires aux narcotiques, anesthésiques ou antiémétiques étant donné la nature et, le moment de la réaction ainsi que la tolérance de ces patients acquise par le passé. Comme les niveaux de méthémoglobine étaient normaux, la méthémoglobinémie a été exclue à son tour. Les auteurs ont jugé peu probable la présence de toxicité sérotoninergique, car la triade de signes et symptômes cliniques n'était pas présente dans tous les cas. Ils ont finalement conclu à une toxicité intrinsèque du $\mathrm{BM}$ et ont recommandé que l'utilisation du BM dans le contexte de parathyroïdectomies soit fortement reconsidérée chez les patients prenant des IRS.

\section{DISCUSSION}

La polémique entourant l'interaction entre le $\mathrm{BM}$ et les IRS demeure d'actualité dans la littérature scientifique. En 

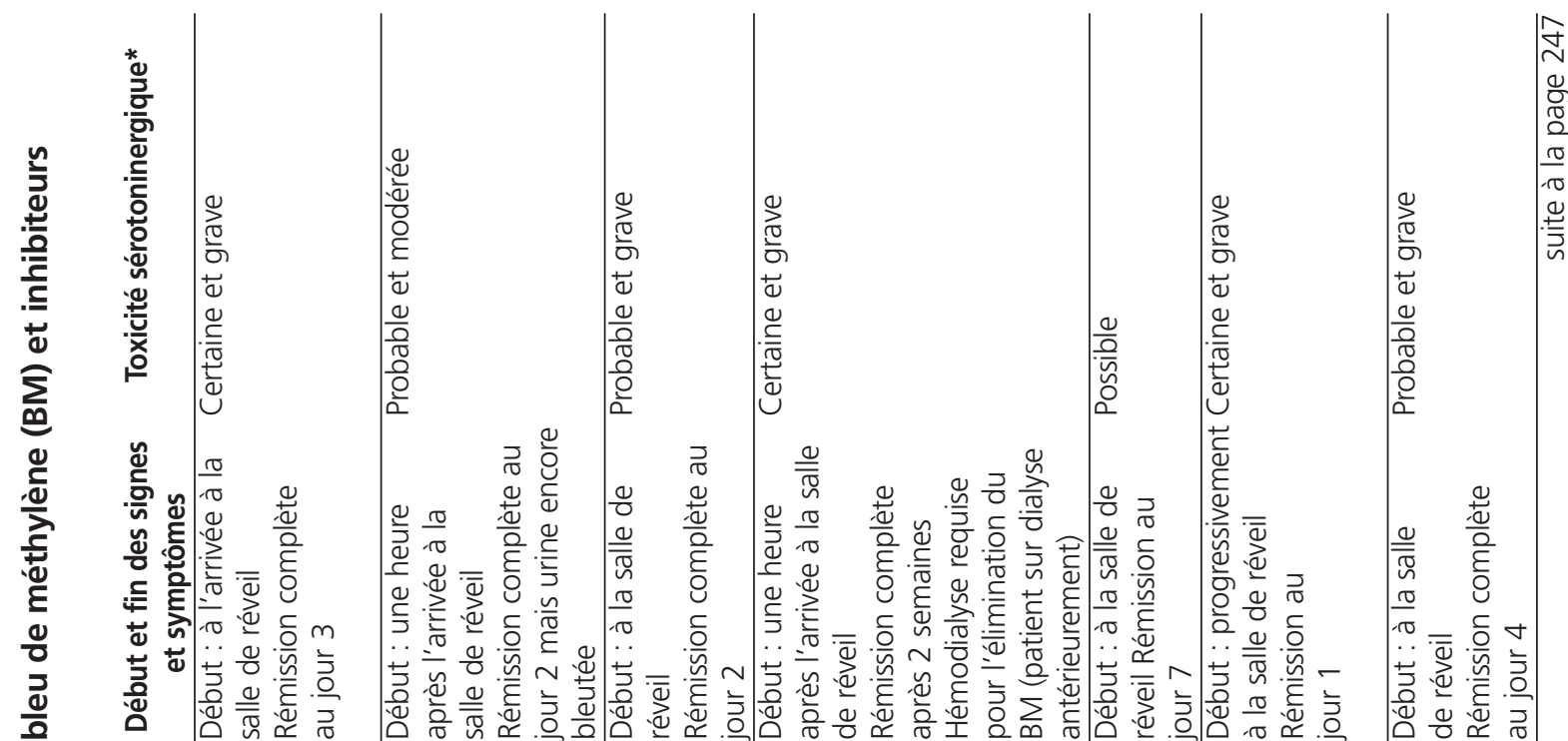

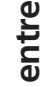
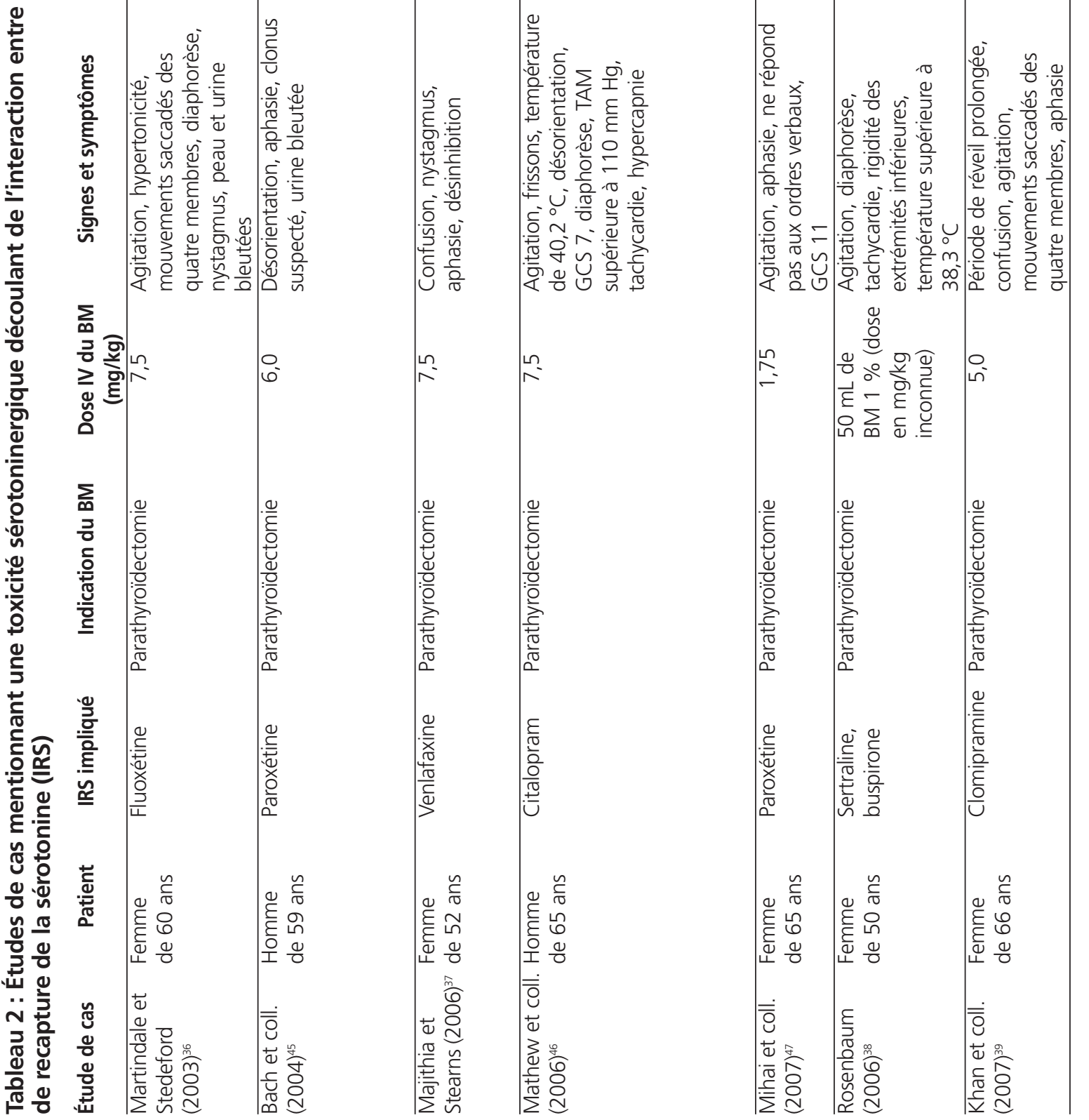

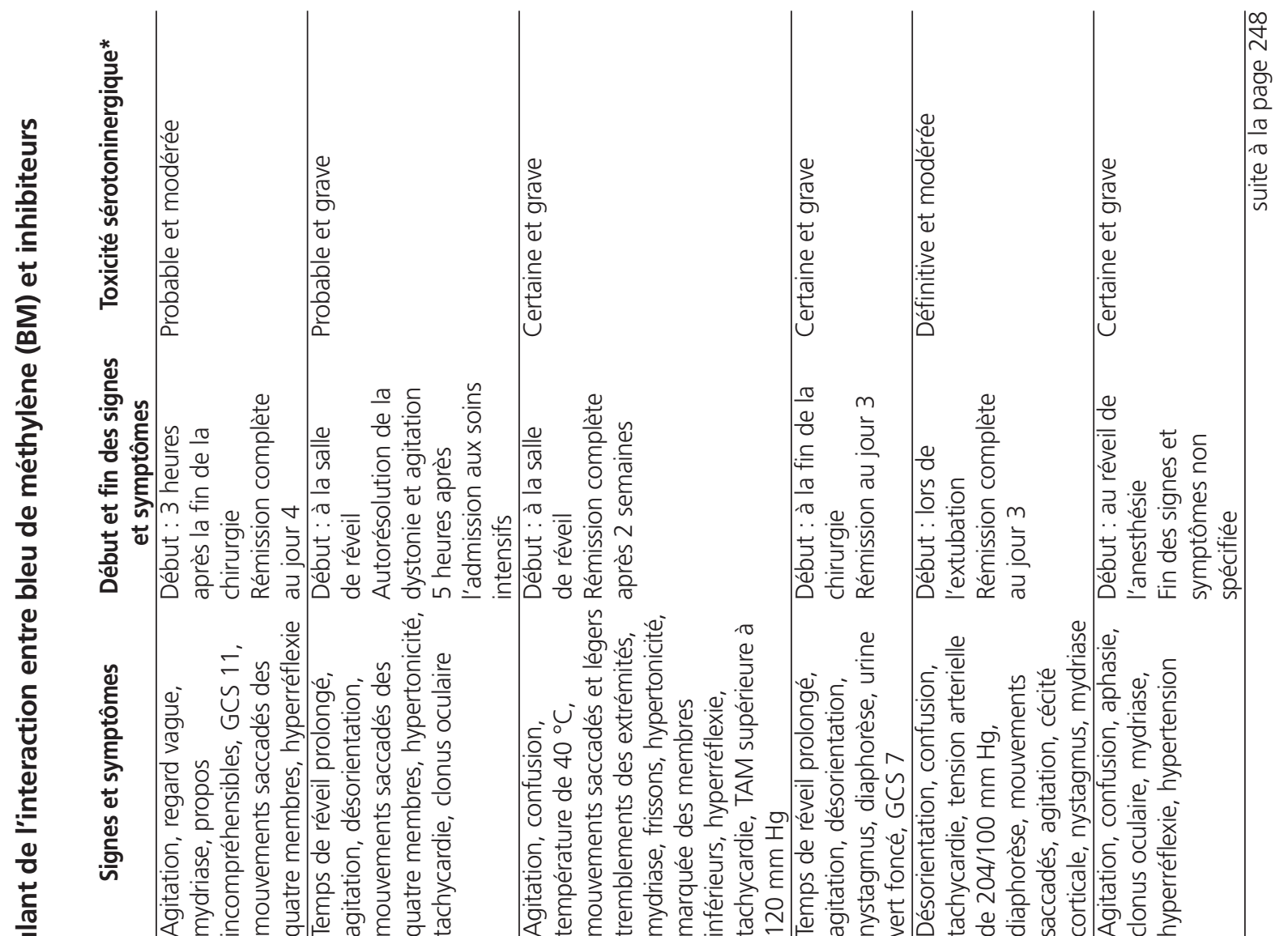

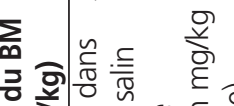
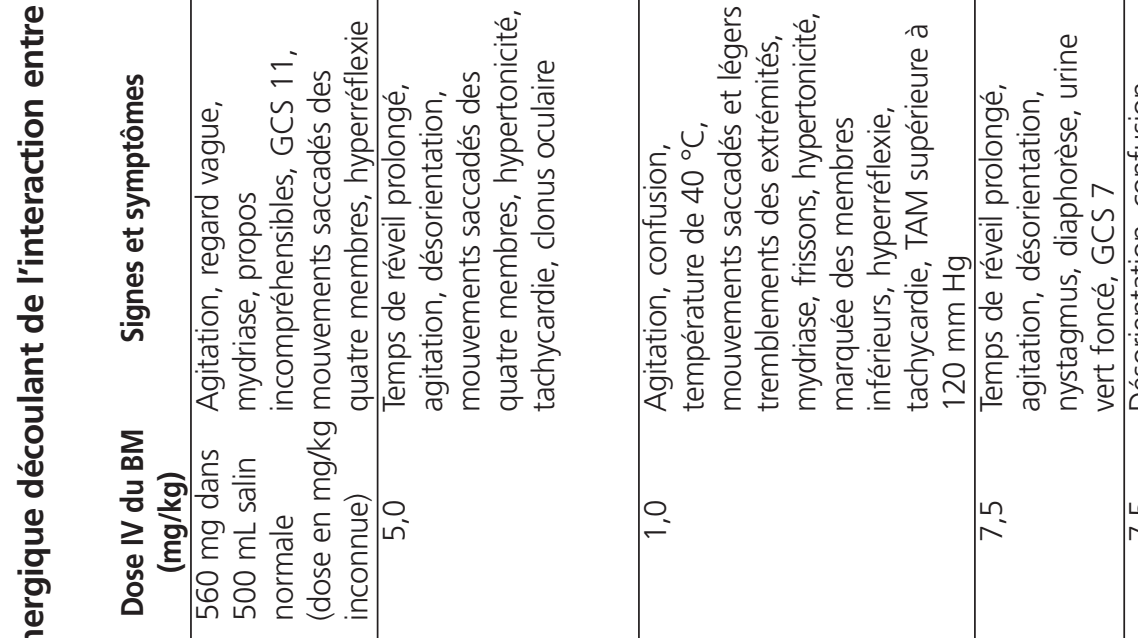

)

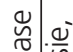

ن.

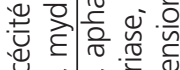

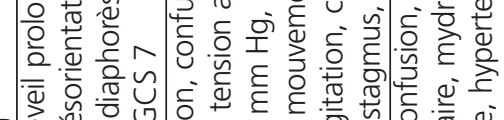

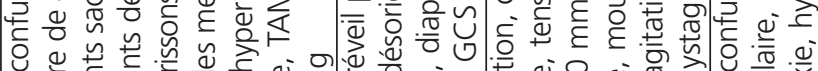

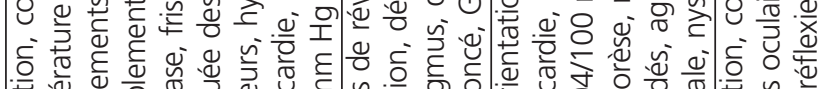

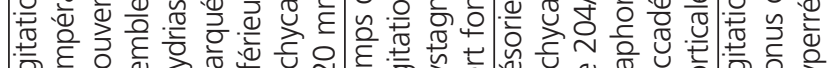

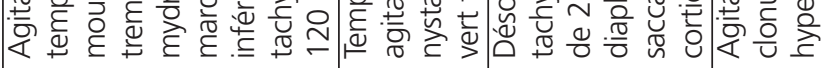

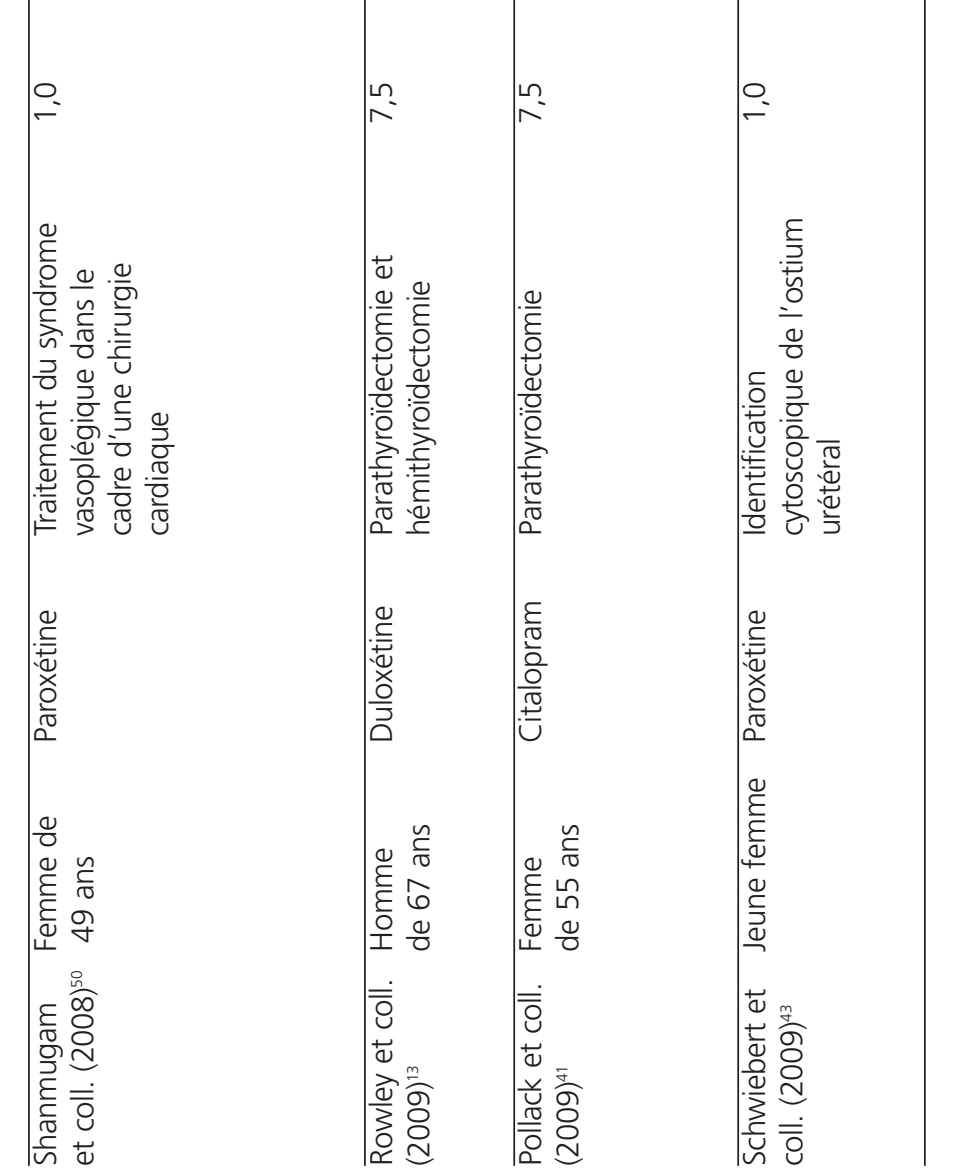




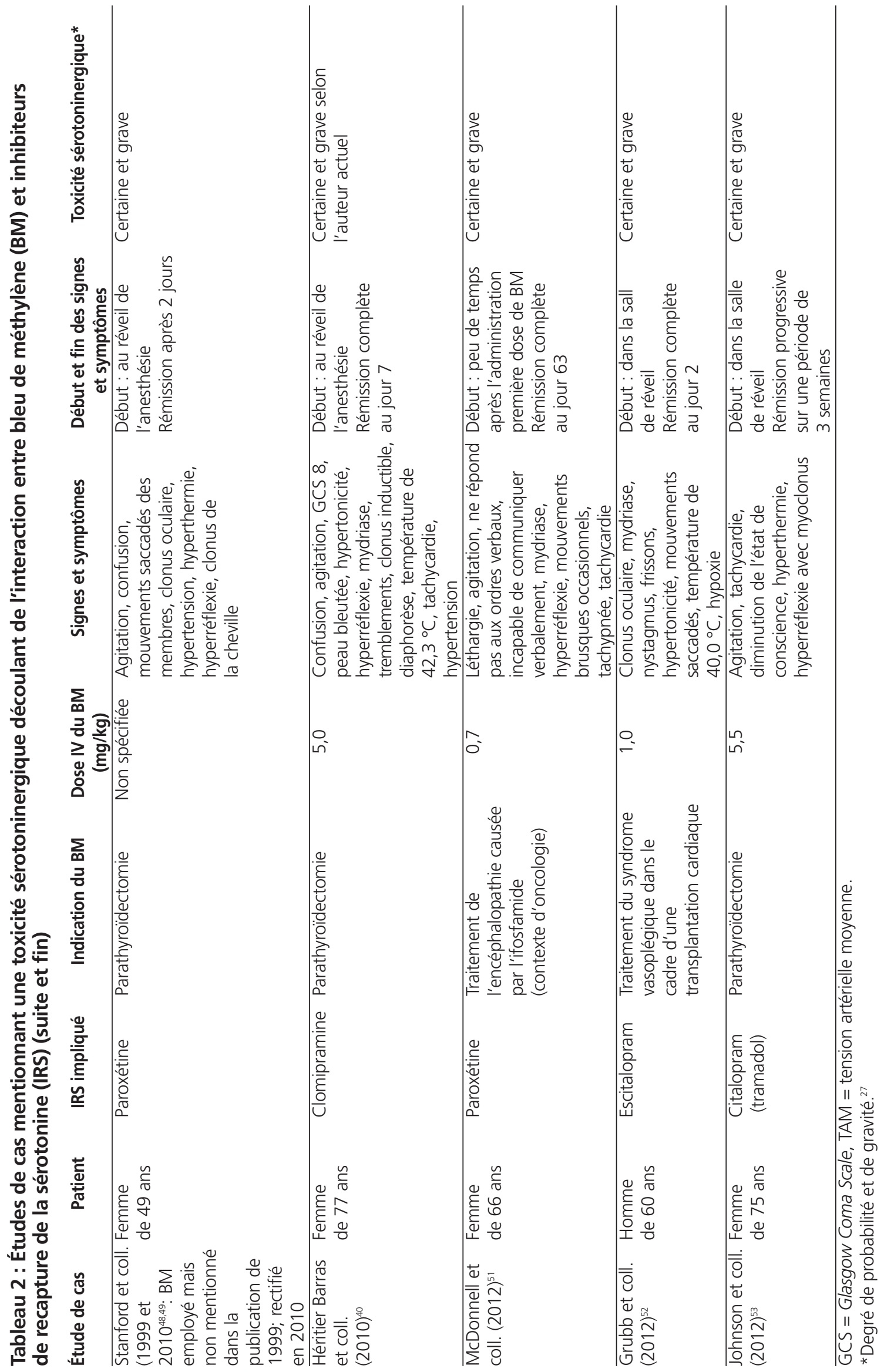


effet, $\mathrm{Ng}$ et $\mathrm{Cameron}^{57}$ ainsi que Gillman ${ }^{27}$ ont chacun publié une revue de littérature portant sur le sujet respectivement en 2010 et 2011. Quant à cette revue, elle présente les données de quatre études de cas supplémentaires, qui ne viennent que confirmer la présence d'interaction significative $e^{40,48,51-53}$.

Bien que cet article comporte plusieurs similitudes avec les deux publications précédentes, il se distingue en ce qui a trait à la méthodologie, aux résultats et à la conclusion. Comparativement aux deux revues de littérature précédentes, la consultation du moteur de recherche Embase s'est révélée nécessaire pour assurer une couverture des publications des journaux européens. De plus, $\mathrm{Ng}$ et Cameron ${ }^{57}$ n'avaient identifié qu'une seule étude de cas dans laquelle le BM avait été employé pour une indication autre que la localisation de la glande parathyroïde. Quant à Gillman, ${ }^{27}$ il avait relevé une étude de cas supplémentaire, où les propriétés colorantes du BM avaient été utilisées dans le cadre d'une procédure chirurgicale autre qu'une parathyrö̈dectomie. Cette revue systématique mentionne deux autres études de cas dans lesquelles le BM a été employé pour une indication autre que celle de la parathyroïdectomie : le traitement du syndrome vasoplégique et le traitement de l'encéphalopathie causée par l'ifosfamide ${ }^{51,52}$. Gillman ${ }^{27}$ avait également conclu que la toxicité sérotoninergique pouvait survenir à des doses de BM aussi faibles que $1 \mathrm{mg} / \mathrm{kg}$ chez les patients prenant un IRS. L'étude de cas de McDonnell et coll. ${ }^{51}$, parue en 2012, révèle que la toxicité sérotoninergique peut se manifester à une dose de $\mathrm{BM}$ aussi faible que $0,7 \mathrm{mg} / \mathrm{kg}$.

Plusieurs hypothèses ont été émises pour expliquer la nature de l'interaction du BM et des IRS. Une première hypothèse repose sur le fait que le BM aurait la capacité de favoriser la libération de la sérotonine par les plaquettes. Cependant, cette première hypothèse ne provient que d'une seule référence datant de $1973^{58}$. Un autre mécanisme, mieux décrit, consisterait en l'inhibition de l'enzyme monoamine oxidase $(\mathrm{MAO})^{59}$. En effet, Ramsay et coll. ont démontré expérimentalement qu'à des concentrations nanomolaires, le $\mathrm{BM}$ est un puissant inhibiteur réversible de la MAO-A, l'enzyme qui inactive principalement la sérotonine et la norépinéphrine dans le cerveau ${ }^{59,60}$. On estime qu'il est un inhibiteur 1000 fois plus puissant mais moins sélectif pour la MAO-A que la moclobémide ${ }^{61}$ Le BM est aussi un inhibiteur de l'enzyme MAO-B mais à des concentrations plus élevées que celles requises pour l'inhibition de la MAO-A ${ }^{60}$. Par contre, il semble que la sérotonine soit peu métabolisée par la MAO-B ${ }^{56,62}$. Ainsi, à des doses cliniques rapportées dans la littérature médicale (de $0,7 \mathrm{mg} / \mathrm{kg}$ à $7,5 \mathrm{mg} / \mathrm{kg}$ ), le $\mathrm{BM}$ administré de façon concomitante avec des IRS conduirait à une inhibition complète de la MAO-A et à une inhibition partielle de la MAO-B, ce qui provoquerait une toxicité sérotoninergique ${ }^{60}$.

Les résultats présentés plus haut semblent appuyer l'hypothèse selon laquelle le BM agirait comme un IMAO.
Tous les cas potentiels de toxicité sérotoninergique sont survenus exclusivement chez des patients prenant un IRS. De plus, la plupart des publications évoquaient des toxicités importantes, ce qui confirmerait le fait que la majorité des toxicités graves soit causée par une interaction impliquant deux agents sérotoninergiques possédant différents mécanismes d'action ${ }^{27}$. Bien que les critères de Hunter soient plus précis et plus spécifiques que ceux de Sternbach pour le diagnostic de la toxicité sérotoninergique, les cas cliniques légers et atypiques ont pu être sous-diagnostiqués ${ }^{20}$. Il faut se rappeler que les critères ont été développés à partir de populations ayant subi une toxicité sérotoninergique résultant d'une surdose d'ISRS et non d'une interaction médicamenteuse ${ }^{22,31}$. Il faut aussi noter que les anesthésiques ont la capacité de supprimer les symptômes-clés de la toxicité sérotoninergique, ce qui aurait pu produire de faux négatifs ${ }^{27}$.

Santé Canada a émis un avis en 2011 à tous les professionnels de la santé concernant la survenue de cas de toxicité sérotoninergique après l'administration de BM IV à des patients prenant des IRS ${ }^{63}$. Plusieurs de ces cas ont nécessité une admission aux soins intensifs. Santé Canada recommande donc fortement que tous les IRS soient cessés sur une période équivalant à quatre à cinq demi-vies d'élimination avant de procéder à l'administration du BM IV.

Quant à la U.S. Food and Drug Administration (FDA), elle a également mis en ligne en 2011 un avertissement concernant le risque de toxicité sérotoninergique lié à l'utilisation du BM en concomitance avec certains médicaments psychiatriques possédant des propriétés sérotoninergiques ${ }^{64}$. Elle mentionne que le $\mathrm{BM}$ ne devrait généralement pas être administré aux patients prenant des IRS, mais elle reconnait toutefois que le recours au BM peut s'avérer nécessaire dans des situations potentiellement fatales, tels la méthémoglobinémie, le traitement de l'encéphalopathie causée par l'ifosfamide ou bien la toxicité au cyanure. Dans un second communiqué, la FDA précise que la plupart des cas de toxicité sérotoninergique ont eu lieu à la suite de l'administration de BM IV dans le cadre de parathyroïdectomies, les doses variant entre $1 \mathrm{mg} / \mathrm{kg}$ et 8 $\mathrm{mg} / \mathrm{kg}^{5}$. Elle évoque le manque de données quant à la survenue de toxicité sérotoninergique lorsque le BM est employé pour d'autres indications ou par d'autres voies d'administration (per os, par injection locale tissulaire). La FDA spécifie par ailleurs dans ce deuxième communiqué que les médicaments psychiatriques sérotoninergiques les plus susceptibles d'induire une toxicité sérotoninergique en présence du BM sont les ISRS, les IRSN et la clomipramine comparativement aux autres antidépresseurs tricycliques, aux IMAO, à la trazodone, à la buspirone, à la mirtazapine et au bupropion pour lesquels l'association avec le risque de toxicité sérotoninergique n'est pas toujours claire. Dans les situations non urgentes, où l'administration du BM est planifiée d'avance, la FDA 
recommande dans tous les cas de cesser les IRS au moins deux semaines avant l'administration du BM. Quant à la fluoxétine, qui possède un temps de demi-vie plus long, l'organisme américain affirme qu'elle devrait être cessée au moins cinq semaines à l'avance ${ }^{64,65}$. La prise des médicaments sérotoninergiques peut être redébutée 24 heures après la dernière dose de BM. Quant aux situations urgentes, le recours à d'autres solutions devrait être étudié avec soin. Si le BM demeure la seule option, les avantages de son administration doivent surpasser les risques. Dans ce cas, l'administration d'IRS devrait être immédiatement interrompue et le patient, suivi étroitement en ce qui a trait à la survenue de signes et de symptômes de toxicité sérotoninergique durant les deux semaines suivant l'administration du BM (ou lors des cinq semaines suivantes pour la fluoxétine) ou jusqu'à 24 heures après la dernière dose de $\mathrm{BM}$ selon l'intervalle de temps qui arrive en premier $^{64}$.

En recommandant d'interrompre l'administration de tout IRS (à l'exception de la fluoxétine) deux semaines avant l'administration du BM, la FDA semble ignorer le risque de provoquer un syndrome de sevrage. Les symptômes de sevrage aux IRS se manifestent notamment par des étourdissements, des nausées, de l'anxiété, de l'agitation, de l'insomnie et de la léthargie. Ces symptômes surviennent habituellement au cours des quatre premiers jours suivant le retrait d'un IRS ou, plus rarement, après la réduction de la dose $e^{66}$. Ainsi, la suspension de l'administration d'un IRS pendant une période de quatre à cinq demi-vies (y compris les métabolites), tel que le suggère Santé Canada, semble suffisante pour prévenir la toxicité sérotoninergique mais aussi pour éviter l'aggravation des symptômes chez la majorité des patients. D'un autre côté, Santé Canada et la FDA ne se sont nullement prononcés sur la possibilité d'une réduction de la dose du BM. Comme la toxicité sérotoninergique semble être un phénomène dépendant de la concentration, cette solution serait fortement envisageable, bien qu'elle n'élimine pas complètement le risque de toxicité sérotoninergique $^{19,31}$. Cela va à l'encontre de l'opinion de Gillman ${ }^{27}$ qui, pour sa part, affirme que la nature de cette interaction est indépendante de la dose, en se basant uniquement sur l'étude de cas de Schwiebert et coll. ${ }^{43}$ dans laquelle on mentionne l'administration d'une dose de $1 \mathrm{mg} / \mathrm{kg}$ de BM.

Dans le cadre du traitement de l'hyperparathyroïdie primaire, plusieurs techniques chirurgicales sont disponibles. Leur utilisation dépend du type de chirurgie à effectuer et de la préférence du chirurgien ${ }^{11}$. On recommande que tous les patients souffrant d'hyperparathyroïdie primaire persistante ou récurrente subissent au moins deux examens non invasifs pour localiser la ou les glandes parathyroïdes anormales, tels le scan à ultrasons et le sestamibi ${ }^{67}$. Si les résultats de ces examens ne concordent pas, il est possible de recourir à la résonance magnétique et au balayage de tomographie calculée. Si l'ensemble des mesures non invasives demeure inefficace, des examens invasifs, telle la mesure de l'hormone parathyroïdienne intraopératoire, peuvent être effectués ${ }^{11,67}$. Cependant, ceux-ci sont moins prisés en raison du risque de complications neurologiques et vasculaires ${ }^{67}$. Quant au BM, son utilisation ne s'appuie sur aucune étude prospective à long terme. Aucune donnée ne permet de croire qu'il diminue le risque d'hyperparathyroïdie primaire persistante ou récurrente ${ }^{11}$. Il n'y a présentement aucune mention dans la littérature médicale d'un agent colorant autre que le BM qui ne soit pas associé à un risque de toxicité sérotoninergique pour les patients prenant des IRS.

Finalement, il n'en demeure pas moins que, la plupart du temps, l'administration du BM échappe à la vigilance du pharmacien à la salle d'opération. À défaut de pouvoir exercer un contrôle rigoureux sur la distribution du BM, l'émission de communiqués et l'organisation de conférences par le département de pharmacie pour sensibiliser les chirurgiens, les intensivistes et les oncologues s'avèrent être un bon début. De plus, la pharmacie pourrait mettre sur pied un document décrivant l'interaction entre le BM et les IRS et fournir une liste de tous les médicaments sérotoninergiques susceptibles d'interagir avec le BM. Ce document pourrait être systématiquement inséré dans tous les dossiers de patients pour qui on planifie une chirurgie ou pour ceux recevant l'ifosfamide.

\section{CONCLUSION}

Jusqu'à présent, 18 études de cas et deux séries de cas ont fait état d'une toxicité sérotoninergique résultant de l'administration du BM à des patients prenant des IRS. Bien que ces toxicités du SNC soient rapportées principalement dans le contexte de parathyroïdectomies, elles sont aussi survenues dans le cadre du traitement du syndrome vasoplégique, de celui de l'encéphalopathie causée par l'ifosfamide et de la localisation de l'ostium urétéral. Le BM agirait comme un inhibiteur de la MAO-A, et ce, à des doses aussi faibles que $0,7 \mathrm{mg} / \mathrm{kg}$. Dans le meilleur des scénarios, l'administration d'IRS devrait être interrompue pendant une période équivalant à quatre à cinq demi-vies avant l'administration du BM. On recommande, quand la cessation de l'IRS est impossible, un suivi étroit des signes et des symptômes de toxicité sérotoninergique.

\section{Références}

1. Oz M, Lorke DE, Hasan M, Petroianu GA. Cellular and molecular actions of methylene blue in the nervous system. Med Res Rev. 2010; 31(1):93-117.

2. Kupfer A, Aeschlimann C, Wermuth B, Cerny T. Prophylaxis and reversal of ifosfamide encephalopathy with methylene-blue. Lancet. 1994;343(8900):763-4.

3. Shanmugam G. Vasoplegic syndrome-the role of methylene blue. Eur J Cardiothorac Surg. 2005;28(5):705-10. 
4. Bradberry SM. Occupational methaemoglobinemia. Mechanisms of production, features, diagnosis and management including the use of methylene blue. Toxicol Rev. 2003;22(1):13-27.

5. FDA drug safety communication: Updated information about the drug interaction between methylene blue (methylthioninium chloride) and serotonergic psychiatric drugs. Silver Spring (MD) : Food and Drug Administration; 2011. Publié au www.fda.gov/Drugs/DrugSafety/ ucm276119.htm. Consulté le 10 septembre 2012.

6. Paciullo CA, McMahon Horner D, Hatton KW, Flynn JD. Methylene blue for the treatment of septic shock. Pharmacotherapy. 2010;30(7): 702-15.

7. Dudley NE. Methylene blue for rapid identification of the parathyroids. Br Med J. 1971;3(5776):680-1.

8. Wheeler $\mathrm{MH}$, Wade JS. Intraoperative identification of parathyroid glands: appraisal of methylene blue staining. Am J Surg. 1982; 143(6):713-6.

9. Gordon DL, Airan MC, Thomas W, Seidman LH. Parathyroid identification by methylene blue infusion. Br J Surg. 1975;62(9):747-9.

10. Derom AF, Wallaert PC, Janzing HM, Derom FE. Intraoperative identification of parathyroid glands with methylene blue infusion. Am J Surg. 1993;165(3):380-2.

11. Harrison BJ, Triponez F. Intraoperative adjuncts in surgery for primary hyperparathyroidism. Langenbecks Arch Surg. 2009;394(5):799-809.

12. Hubbard JGH, Inabnet WB, Lo CY, rédacteurs. Endocrine surgeries: principles and practice. Springer; 2009. pages 270-271. Publié au http://books.google.ca/books?id=mYr3ojk3ggEC\&dq=methylene+blue+ dye+administration+pARATHYROID\&hl=fr\&source=gbs_navlinks_s. Consulté le 22 août 2012.

13. Rowley M, Riutort K, Shapiro D, Casler J, Festic E, Freeman WD. Methylene blue-associated serotonin syndrome: a green encephalopathy after parathyroidectomy. Neurocrit Care. 2009;11(1):88-93.

14. Waye JD. Advanced polypectomy. Gastrointest Endosc Clin N Am. 2005;15(4):733-56.

15. Munakata A, Uno Y. Colonoscopic polypectomy with local injection of methylene blue. Tohuku J Exp Med. 1994;173(4):377-82.

16. Wainwright M, Crossley KB. Methylene blue: a therapeutic dye for all seasons? J Chemother. 2002;14(5):431-43.

17. Nadler JE, Green H, Rosenbaum A. Intravenous injection of methylene blue infusion in man with reference to its toxic symptoms and effect on electrocardiogram. Am J Med Sci. 1934;188:15-21.

18. Iqbal MM, Basil MJ, Kaplan J, Iqbal MT. Overview of serotonin syndrome. Ann Clin Psychiatry. 2012;24(4):310-8.

19. Gillman PK. A review of serotonin toxicity data: implications for the mechanisms of antidepressant drug action. Biol Psychiatry. 2006;59(11): 1046-51.

20. Boyer EW, Shannon M. The serotonin syndrome. $N$ Engl J Med. 2005;352(11):1112-20.

21. Sun-Edelstein C, Tepper SJ, Shapiro RE. Drug-induced serotonin syndrome: a review. Expert Opin Drug Saf. 2008;7(5):587-96.

22. Isbister GK, Buckley NA, Whyte IM. Serotonin toxicity: a practical approach to diagnosis and treatment. Med J Aust. 2007;187(6):361-5.

23. Mason PJ, Morris VA, Balcezak TJ. Serotonin syndrome: presentation of 2 cases and review of the literature. Medicine (Baltimore). 2000; 79(4):201-9.

24. Isbister, GK, Hackett LP, Dawson AH, Whyte IM, Smith AJ. Moclobemide poisoning: toxicokinetics and occurrence of serotonin toxicity. Br J Clin Pharmacol. 2003;56(4):441-50.

25. Isbister GK, Bowe SJ, Dawson A, Whyte IM. Relative toxicity of selective serotonin reuptake inhibitors (SSRIs) in overdose. J Toxicol Clin Toxicol. 2004; 42(3):277-85.

26. Lam SW, Engebretsen KM, Bauer SR. Toxicology today: what you need to know now. J Pharm Pract. 2011;24(2):174-88.

27. Gillman PK. CNS toxicity involving methylene blue: the exemplar for understanding and predicting drug interactions that precipitate serotonin toxicity. J Psychopharmacol. 2011;25(3):429-36.

28. Mohammad-Zadeh LF, Moses L, Gwaltney-Brant SM. Serotonin: a review. J Vet Pharmacol Ther. 2008;31(3):187-199.
29. Martin T. Serotonin syndrome. Ann Emerg Med. 1996;28(5):520-6.

30. Gillman PK. The serotonin syndrome and its treatment. J Psychopharmacol. 1999;13(1):100-9.

31. Dunkley EJC, Isbister GK, Sibbritt D, Dawson AH, Whyte IM. The Hunter serotonin toxicity criteria: simple and accurate diagnostic decision rules for serotonin toxicity. QJM. 2003;96(9):635-42.

32. Isbister GK, Buckley NA. The pathophysiology of serotonin toxicity in animals and humans: implications for diagnosis and treatment. Clin Neuropharmacol. 2005;28(5):205-14.

33. Patel AS, Singh-Ranger D, Lowery KA, Crinnion JN. Adverse neurologic effect of methylene blue used during parathyroidectomy. Head Neck. 2006;28(6):567-8.

34. Hajj-Chahine J, Jayle C, Corbi P. Methylene blue in patients with severe pulmonary hypertension. J Thorac Cadiovasc Surg. 2013;145(3):898.

35. Mulroy E, Farrell M, Lynch T. Serotonin syndrome: three case reports and pathological findings [résumé]. Mov Disord. 2012;27 Suppl 1:1297.

36. Martindale SJ, Stedeford JC. Neurological sequelae following methylene blue injection for parathyroidectomy. Anaesthesia. 2003;58(10):1041-2.

37. Majithia A, Stearns MP. Methylene blue toxicity following infusion to localize parathyroid adenoma. J Laryngol Otol. 2006;120(2):138-40.

38. Rosenbaum HK. Case of the month: thyroidectomy—postop agitation and rigidity; serotonin syndrome. janvier 2006. Publié au http:// medical.mhaus.org/index.cfm/fuseaction/Content.Display/PagePK/ CaseOfTheMonth2.cfm. Consulté le 3 septembre 2012.

39. Khan MAS, North AP, Chadwick DR. Prolonged postoperative altered mental status after methylene blue infusion during parathyroidectomy: a case report and review of the literature. Ann R Coll Surg Engl. 2007; 89(2):W9-11.

40. Héritier Barras AC, Walder W, Seeck M. Serotonin syndrome following methylene blue infusion: a rare complication of antidepressant therapy. J Neurol Neurosurg Psychiatry. 2010;81(12):1412-3.

41. Pollack G, Pollack A, Delfiner J, Fernandez J. Parathyroid surgery and methylene blue: a review with guidelines for safe intraoperative use. Laryngoscope. 2009;119(10):1941-6.

42. Ng BKW, Cameron AJD, Liang R, Rahman H. Serotonin syndrome following methylene blue infusion during parathyroidectomy: a case report and literature review. Can J Anaesth. 2008;55(1):36-41.

43. Schwiebert C, Irving C, Gillman PK. Correspondence: Small doses of methylene blue, previously considered safe, can precipitate serotonin syndrome. Anaesthesia. 2009;64(8):924.

44. Khavandi A, Whitaker J, Gonna H. Serotonin toxicity precipitated by concomitant use of citalopram and methylene blue. Med J Aust. 2008;189(9):534-5.

45. Bach KK, Lindsay FW, Berg LS, Howard RS. Prolonged postoperative disorientation after methylene blue infusion during parathyroidectomy. Anesth Analg. 2004;99(5):1573-4.

46. Mathew S, Linhartova L, Raghuraman G. Hyperpyrexia and prolonged postoperative disorientation following methylene blue infusion during parathyroidectomy. Anaesthesia. 2006;61(6):580-3.

47. Mihai R, Mitchell EW, Warwick J. Dose-response and postoperative confusion following methylene blue infusion during parathyroidectomy. Can J Anaesth. 2007;54(1):79-81.

48. Stanford SC, Stanford BJ, Gillman PK. Risk of severe serotonin toxicity following co-administration of methylene blue and serotonin reuptake inhibitors: an update on a case report of post-operative delirium. $J$ Psychopharmacol. 2010;24(10):1433-8.

49. Stanford BJ, Stanford SC. Postoperative delirium indicating an adverse drug interaction involving the selective serotonine reuptake inhibitor, paroxetine? J Psychopharmacol. 1999;13(3):313-7.

50. Shanmugam G, Kent B, Alsaiwadi T, Baskett R. Serotonin syndrome following cardiac surgery. Interact Cardiovasc Thorac Surg. 2008;7(4):656-7.

51. McDonnell AM, Rybak I, Wadleigh M, Fisher DC. Suspected serotonin syndrome in a patient being treated with methylene blue for ifosfamide encephalopathy. J Oncol Pharm Pract. 2012;18(4):436-9.

52. Grubb KJ, Kennedy JLW, Bergin JD, Groves DS, Kern JA. The role of methylene blue in serotonin syndrome following cardiac transplantation: a case report and review of the literature. J Thorac Cardiovasc Surg. 2012;144(5):e113-6. 
53. Johnson N, Soar J, Robinson S. Concurrent administration of methylene blue and a selective serotonin reuptake inhibitor: a recipe for serotonin syndrome. J Intens Care Soc. 2012;13(3):256-8.

54. Sweet G, Standiford SB. Methylene-blue-associated encephalopathy. J Am Coll Surg. 2007;204(3):454-8.

55. Kartha SS, Chacko CE, Bumpous JM, Fleming M, Lentsch EJ, Flynn MB. Toxic metabolic encephalopathy after parathyroidectomy with methylene blue localization. Otolaryngol Head Neck Surg. 2006; 135(5):765-8.

56. Craig CR, Stitzel RE, rédacteurs. Modern pharmacology with clinical applications. $5^{\circ}$ édition. Boston (MA) : Lippincott Williams \& Wilkins; 1997. pages 356, 392.

57. $\mathrm{Ng}$ BKW, Cameron AJD. The role of methylene blue in serotonin syndrome: a systematic review. Psychosomatics. 2010;51(3):194-200.

58. Schick PK, Yu BP. Methylene blue-induced serotonin release in human platelets. J Lab Clin Med. 1973;82(4):546-53.

59. Aeschlimann C, Cerny T, Küpfer A. Inhibition of (mono)amine oxidase activity and prevention of ifosfamide encephalopathy by methylene blue. Drug Metab Dispos. 1996;24(12):1336-9.

60. Ramsay RR, Dunford C, Gillman PK. Methylene blue and serotonin toxicity: inhibition of monoamine oxidase A (MAO A) confirms a theoretical prediction. Br J Pharmacol. 2007;152(6):946-51.

61. Ulus IH, Maher TJ, Wurtman RJ. Characterization of phentermins and related compounds as monoamine oxidase (MAO) inhibitors. Biochem Pharmacol. 2000;59(12):1611-21.

62. Ochiai Y, Itoh K, Sakurai E, Adachi M, Tanaka Y. Substrate selectivity of monoamine oxidase $\mathrm{A}$, monoamine oxidase $\mathrm{B}$, diamine oxidase, and semicarbazide-sensitive amine oxidase in COS-1 expression systems. Biol Pharm Bull. 2006;29(12):2362-6.

63. Avis aux hôpitaux: Renseignements importants en matière d'innocuité émis par Santé Canada concernant le bleu de méthylène injectable. Ottawa (ON) : Santé Canada; 2011. Publié au www.hc-sc.gc.ca/ dhp-mps/medeff/advisories-avis/prof/_2011/methylene_blue-bleu_ nth-aah-fra.php. Consulté le 10 septembre 2012.
64. FDA drug safety communication: serious CNS reactions possible when methylene blue is given to patients taking certain psychiatric medications. Silver Spring (MD) : U.S. Food and Drug Administration; 2011. Publié au www.fda.gov/Drugs/DrugSafety/ucm263190.htm. Consulté le 10 septembre 2012.

65. Isbister GK, Dawson A, Whyte IM, Prior FH, Clancy C, Smith AJ. Neonatal paroxetine withdrawal syndrome or actually serotonin syndrome? Arch Dis Child Fetal Neonatal Ed. 2001;85(2):F147-8.

66. Haddad PM, Anderson IM. Recognising and managing antidepressant discontinuation. Adv Psychiatr Treat. 2007;13:447-57.

67. Caron NR, Sturgeon C, Clark OH. Persistent and recurrent hyperparathyroidism. Curr Treat Options Oncol. 2004;5(4):335-45.

Annie Charbonneau, B. Pharm, M. Sc., est pharmacienne ayant pour secteurs de pratique la médecine interne, la gériatrie ainsi que le Centre d'Information pharmacothérapeutique, Centre universitaire de santé McGill (CUSM), Montréal, Québec.

Intérêts concurrents : aucun déclaré.

Adresse de correspondance :

Annie Charbonneau

Hôpital Royal Victoria

687, avenue Pine Ouest

Montréal QC H3A 1A1

Courriel : annie.charbonneau@muhc.mcgill.ca

\section{Remerciements}

L'auteur voudrait remercier madame Marjorie Friesen (pharmacienne à I'Hôpital Royal Victoria) ainsi que monsieur William Poggemiller (étudiant au Pharm. D. à I'Université de Toronto au moment de la rédaction du texte) pour leurs judicieux conseils et encouragements.

\section{Validity Evidence for FASTHUG-MAIDENS, a Mnemonic for Identifying Drug-Related Problems in the Intensive Care Unit: Correction}

The Acknowledgements section was inadvertently omitted from the recently published article presenting validity evidence for the FASTHUG-MAIDENS mnemonic. ${ }^{1}$ The acknowledgements are presented here:

We acknowledge the contributions of the following individuals to our work: Chris Lo, PharmD (Clinical Pharmacy Practice Leader, Langley Memorial Hospital), and Bob Gillies, PharmD (Clinical Pharmacy Specialist-ICU, Surrey Memorial Hospital), who acted as ICU pharmacists; Mike Wasdell, a statistician formerly with the Department of Evaluation and Research Services, Fraser Health; and the pharmacy residents who participated in data collection.

\section{Reference}

1. Masson SC, Mabasa VH, Malyuk DL, Perrott JL. Validity evidence for FASTHUG-MAIDENS, a mnemonic for identifying drug-related problems in the intensive care unit. Can J Hosp Pharm. 2013;66(3):157-62. 